\title{
Human enhancement: could you become infected with a computer virus?
}

Conference or Workshop Item

Published Version

Gasson, M. N. (2010) Human enhancement: could you become infected with a computer virus? In: 2010 IEEE International Symposium onTechnology and Society (ISTAS), 7-9 June 2010, Wollongong, Australia, pp. 61-68. Available at http://centaur.reading.ac.uk/29329/

It is advisable to refer to the publisher's version if you intend to cite from the work. See Guidance on citing.

Published version at: http://dx.doi.org/10.1109/ISTAS.2010.5514651

All outputs in CentAUR are protected by Intellectual Property Rights law, including copyright law. Copyright and IPR is retained by the creators or other copyright holders. Terms and conditions for use of this material are defined in the End User Agreement. 


\section{CentAUR}

Central Archive at the University of Reading

Reading's research outputs online 


\title{
Human Enhancement: Could you become infected with a computer virus?
}

\author{
Dr. Mark N. Gasson \\ School of Systems Engineering, University of Reading, UK \\ m.n.gasson@reading.ac.uk
}

\begin{abstract}
Experiments demonstrating human enhancement through the implantation of technology in healthy humans have been performed for over a decade by some academic research groups. More recently, technology enthusiasts have begun to realize the potential of implantable technology such as glass capsule RFID transponders. In this paper it is argued that implantable RFID devices have evolved to the point whereby we should consider the devices themselves as simple computers. Presented here is the infection with a computer virus of an RFID device implanted in a human. Coupled with our developing concept of what constitutes the human body and its boundaries, it is argued that this study has given rise to the world's first human infected with a computer virus. It has taken the wider academic community some time to agree that meaningful discourse on the topic of implantable technology is of value. As developments in medical technologies point to greater possibilities for enhancement, this shift in thinking is not too soon in coming.
\end{abstract}

\section{Introduction}

Is the human body a suitable place for a microchip? Such discussion is no longer hypothetical - in fact in reality it has not been so for some years. Although the step from pervasive to physically invasive may seem a long one, the transition is blurred. Medical devices such as pacemakers and cochlear implants have become well established, yet these sophisticated devices form notably intimate links between technology and the body. More recent developments in engineering technologies have meant that the ability to integrate silicon with biology is reaching new levels. Widely publicized experiments conducted at the University of Reading to surgically implant technologies have already taken steps forward by linking the human nervous system directly to a computer, people are opting to have passive silicon devices surgically implanted to allow identification and tracking and medical devices which interact directly with the brain (e.g. deep brain stimulators for the treatment of Parkinson's disease) are becoming commonplace.
The application of implantable technology for medical use is typically 'restorative', i.e. it aims to restore some deficient ability. However, as these medical technologies continue to advance, their potential benefits for human enhancement, i.e. enabling abilities over and above those which humans normally possess, will become increasingly attractive. It is therefore important that we seriously consider where this may take us. Scientists have indicated for some time that a human/machine symbiosis - a physical linking of the two entities such that humans can seamlessly harness the power of machine intelligence and technological capability - is a real possibility. While it is necessary to acknowledge that our continuing evolution may well mean that we all become part machine, we must also be mindful of the new threats this step brings. It is clear that a number of issues stem from such enhancement and it is timely to have debate to address the wider implications.

\section{Human enhancement through implantable technology}

On Monday, August 24th, 1998, a groundbreaking experiment was conducted by Professor Warwick's group at the University of Reading in the UK. At the heart of this work was the sub-dermal implantation of a Radio Frequency IDentification (RFID) $\operatorname{tag}^{1}$ and the augmentation of the infrastructure at the university's Department of Cybernetics with RF nodes such that the system was able to track Warwick, via the tag, as he roamed the building. The possibilities using this technology were, even at that time, not greatly limited, although the system was restricted to simple profiling of his behavior. From this, automated customization of his environment was possible, such as unlocking doors, turning on lights and brewing his coffee on arrival.

While the public response to this work was varied, from suggestions that this was the work of the devil, ${ }^{2}$ to

\footnotetext{
${ }^{1}$ In brief, RFID tags wirelessly communicate data to reader devices from which typically the power is supplied wirelessly to the tag. The data, in the simplest devices, is a unique code which identifies the tag, and thus the object, if known, to which it is attached.

${ }^{2}$ Revelation 13:16-18 "He [the beast] also forced everyone, small and great, rich and poor, free and slave, to receive a mark on his right hand
} 
awe of the technological possibilities, acknowledgement of the prophetic merit largely mirrored that of academic musings on the scientific value. Few people would entertain the idea that people may actually be open to having such devices implanted if there was some net benefit in doing so. Equally, many people were unaware that, at that time, passive RFID tag technology was on the cusp of becoming cost effective enough to essentially become ubiquitous.

Some six years later, implantable identifying RFID tags were commercialized by 'VeriChip' and approved by the FDA in the USA for human use. It was proposed that these devices could essentially replace 'medic alert' bracelets by linking a person to their medical details in an online medical database. Such devices have subsequently been used to allow access to secure areas in building complexes, for example the Mexican Attorney General's office implanted 18 of its staff members in 2004 to control access to a secure data room, and nightclubs in Barcelona, Spain and The Netherlands use the VeriChip implant to allow entry to their VIP customers, and enable automated payments. By 2005, reports of people implanting themselves with commercially available RFID tags for a variety of applications had become a familiar if not regular occurrence (see e.g. [1]).

The broad discussion on security and privacy issues regarding mass RFID deployment has gathered momentum, and security experts are now specifically warning of the inherent risks associated with using RFID for the authentication of people, see [2] for an overview. Whilst the idea that RFID can be used to covertly track an individual 24-7 betrays a fundamental misunderstanding of the limitations of the technology, there are genuine concerns to address. The use of RFID implants is especially thwart with issues because being implanted forms a clear, permanent link with the individual and makes compromised devices hard to revoke. Because of this, concerns for those who have decided to have an RFID tag implanted are valid. It is however assumed that such procedures will never become compulsory and so most people will remain unaffected. However, while mass deployment of RFID technologies is well documented, especially in the context of commerce such as supply chain management, it should be noted that, through nonnefarious means, it is possible that people could become implanted with RFID technology unknowingly. This is mostly related to safety issues regarding passive medical devices such as hip replacements and breast implants whereby being able to determine the exact manufacturing details non-invasively could be advantageous. This is especially valuable when manufacturing faults are

or on his forehead, so that no one could buy or sell unless he had the mark, which is the name of the beast or the number of his name". Such scaremongering is in keeping with the flawed logic which demonstrates that the common Universal Product Code contains a hidden ' 666 '. subsequently discovered and devices of unknown provenance have been used. Embedding RFID technology in a device before it is surgically utilized enables this function. Further, following the polemic on silicone-gel breast implants [3], a device based around RFID technology, designed to be located inside the breast, which detects rupture has been developed. The benefits of being able to non-invasively monitor the condition of a medical device, such as a heart valve, using this type of technology are also being investigated. However, all of these applications result in the wider issues of having RFID technology implanted.

Exact numbers of those who have implanted RFID technology are not known, but it is clear that the figure is rising, and, with familiarity, public acceptance will surely grow. Because we largely dismissed such uses of the technology as improbable some ten years ago, a lack of timely debate on the wider implications means that we are now faced with the prospect of addressing them whilst the technology gets a foot-hold. It is not hard to imagine that dealing with technical and wider issues retrospectively will be immensely more difficult.

Having seen the applications which RFID has found despite earlier pessimism, we should consider the application of the more advanced medically orientated technologies on healthy individuals, i.e. enhancement rather than restoration, as a distinct probability.

\section{Medical application of implantable technology}

There is a fair range of 'restorative' devices already in clinical use, although many, such as artificial joints, only have simple mechanical function. Other devices, such as the artificial heart pacemaker, have become notably sophisticated in recent years with integrated movement sensors to adjust heart rate based on estimated demand, internal logging of biological data, and $R F$ communication with the outside world. In the main, society has come to accept restorative technologies such as pacemakers, although these devices have become advanced in their function to the point of causing security and privacy concerns [4].

Of great interest is the development of technologies which are able to interact with us on a neural level. The most ubiquitous sensory neural prosthesis is by far the cochlear implant [5]. With the limitations of the cochlear implant in mind, the artificial visual prosthesis [6] is certainly substantially more ambitious. While both cochlear implants and retina stimulators operate by artificially manipulating the peripheral nervous system, less research has been conducted on direct electrical interaction with the human central nervous system, and in particular the brain. Work on animals [7], [8] has 
demonstrated how direct brain stimulation can be used to guide rats through a maze problem, essentially by reinforcement, by evoking stimuli to the cortical whisker areas to suggest the presence of an object, and stimulation of the medial forebrain bundle (thought to be responsible for both the sense of motivation and the sense of reward) when the rat moves accordingly. Early work to translate this research to humans demonstrated radical (and occasionally dubiously interpreted) changes in mood and personalities when such 'pleasure centers' were stimulated [9], [10]. This period saw some seventy patients implanted with permanent micro-stimulators to treat a variety of disorders with reportedly good success, although the indiscriminant use of the procedure and significant failure rate saw it largely condemned. This may have been partly because the disorders targeted were psychiatric rather than neurologic [11].

It was not until the 1980s, when French scientists discovered that the symptoms of Parkinson's disease (PD), with better understood anatomical pathology, were treatable using Deep Brain Stimulation (DBS), that research again picked up pace. However, difficulties in accurately targeting structures deep in the brain, lack of safe durable electrodes, problems of miniaturizing electronics and power supply limitations meant that such therapy was not readily available for several more years.

Recently there has been a resurgence of interest in the surgical treatment of movement disorders such as PD. This is because of the disabling side effects of long term treatment with L-dopa ${ }^{3}$. Also many movement disorders, such as multiple system atrophy or dystonia, do not respond to dopaminergic treatment at all. A limited range of DBS systems have been made commercially available and are now in clinical use despite their significant cumulative costs, largely due to repeat operations to replace exhausted battery packs [12], and known neuropsychological side effects [13].

The ability of electrical neural stimulation to drive behavior and modify brain function without the recipient's cognitive intervention is evident from this type of device. Furthermore, it has been demonstrated how electrical stimulation can be used to replace the natural percept, for example the work by Romo et al. [14]. However, in all cases these devices operate in a unidirectional fashion - the ability to form direct bidirectional links with the human nervous system certainly opens up the potential for many new application areas. However, bi-directional neural implants are very much experimental. Whilst they have much potential in the areas of prosthetics, major developments have been slow in coming. Recent research in the area of DBS has shown

\footnotetext{
${ }^{3}$ L-dopa is a chemical precursor to dopamine which can cross the blood-brain barrier and metabolize in the brain to address insufficient dopamine levels, thought to be a primary cause of PD.
}

that by recording brain activity via the implanted electrodes it is possible to detect characteristic signal changes in the target nuclei prior to the event of tremor, and so stimulation based on a prediction of what the brain will do is possible [15]. The development of such technologies, which are able to decode the brain's function and operate bi-directionally, is clearly of great value.

\section{Where Restorative meets Enhancement}

The relatively new trend for having passive RFID implants has recently risen in the public consciousness, although less publicized developments of high-tech implants in the medical domain have been progressing for several decades. Indeed, a significant drive behind the development of implantable devices is medical - i.e. restoring deficient abilities in humans. Given this, there are two clear routes by which technology developed for restorative application may ultimately lead to enhancement. The first is that it is conceivable that a piece of technology designed as a restorative device may actually give the recipient a capability which exceeds the normal human ability it is designed to replace. For example, advances in cochlear implants may result in the recipient having vastly improved hearing over that of a normal human which could then be considered enhancement. The discussion in this context has begun on the topic of prosthetic limbs [16]. There are however no clear examples relating to implantable technology to date, although Moore [17] describes the case of a patient with an artificial heart who found he could use the device to lower his heart rate to help falling asleep.

The second is the application of implantable technology, developed initially in a medical context, to augment the abilities of healthy humans. Reports of this pioneering step are rare, although in a notable echo of 1998, the University of Reading in the UK has been active in this area. On March 14th, 2002, an array of one hundred individual needle electrodes was surgically implanted into the median nerve fibers of the left arm of Professor Kevin Warwick, a healthy volunteer [18], [19] (see also [20] for a personal account). This study demonstrated, in a rudimentary fashion, a range of applications, from nervous system to nervous system communication, feedback control of robotic devices and augmented sensory capabilities.

To date there are no studies involving implantation in the central nervous system of healthy volunteers that have been well reported. There is, however, some largely anecdotal evidence of the occasional positive side effect that Deep Brain Stimulation (DBS) in patients has had. In one such case, a graphic designer, who received DBS surgery for a severe Tourettes disorder, found that stimulation through one specific electrode could actually 
make her more creative. Indeed, when this electrode was used, her employer noted an improvement in color and layout in her graphic design work [21]. The application of this type of effect in the long term clearly cannot be discounted, and so nor can the translation of medical devices to enhancement. Indeed, the ability to form direct, bi-directional links with the human brain will open up the potential for many new application areas. While still in its infancy, scientists predict that within the next thirty years neural interfaces will be designed that will not only increase the dynamic range of senses, but may also enhance memory and enable "cyberthink" - invisible communication with others and technology [22].

\section{The human body and the computer virus}

It is evident that advances in technology, coupled with a change of perception and attitudes within society, partly driven by increasing familiarity, are enabling new opportunities for human enhancement. While the willingness of self-experimenters to push the boundaries is of great importance, it is the gradual evolution of technology which enables notable change. Mobile phone technology, for example, has gone from bulky phone only handsets to complex multi-functional 'smart phone' devices in a matter of years. Indeed these devices are no longer simply mobile phones - they are now more like mobile computers on which we can make phone calls.
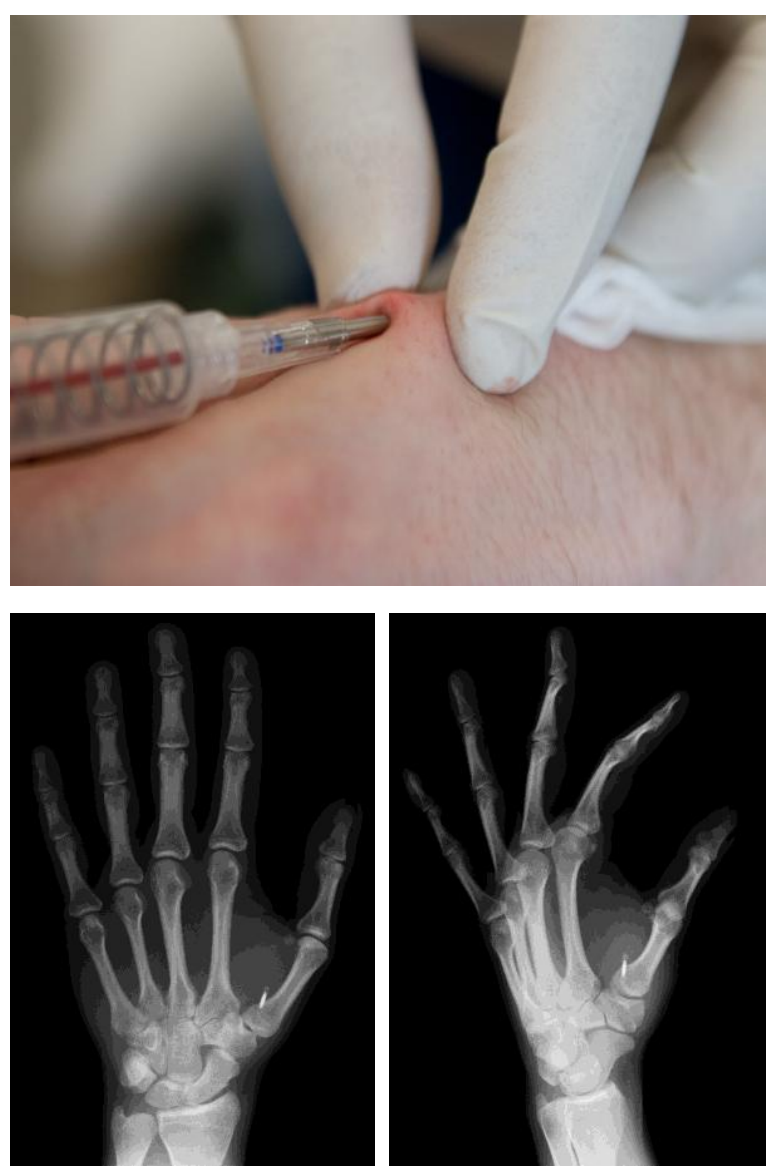

Figure 1. An RFID tag is injected into the left hand of the author by a surgeon (left), shown in close up (top right). Two x-ray images taken post-procedure (bottom right) show the position of the tag in the hand near the thumb

It seems that mobile handsets are the first wave of successful 'wearable computers' prophesized for years by scientists [23], at least in the sense that they comprise a relatively powerful computing device which people habitually carry with them, if not 'wear' as such. These devices are viewed as the forerunner to 'ubiquitous computing' and 'Ambient Intelligent Environments', other paradigm shifts predicted in our evolving relationship with technology [24]. Certainly this is not what was originally envisaged. With this in mind, the case of RFID tags is an interesting one. In their early application as an implantable device they had very simple functionality - the ability to broadcast a fixed unique identifier over a short range on request. While largely deployed for animal identification, the implantable tags commercialized for human use had the same function - an identifier which could be cross-referenced with a database that held all other information. However, the core 
technology has continued to develop, and although nonimplantable RFID devices in general remain more advanced than implantable, glass capsule types, these too continue to evolve which opens up new possibilities, and new issues. To further explore this, the earliest experiments with an implanted RFID device conducted in 1998 have been revisited using the latest in implantable RFID technology.

On the $16^{\text {th }}$ March 2009 , in a single participant study, the author of this paper had a glass capsule HITAG S 2048 RFID device implanted into his left hand (see Figure 1). While containing a 32-bit unique identifier number, similarly to older devices, the device also has a 2048-bit $\mathrm{read} /$ writable memory to store data and the option of 48bit secret key based encryption for secure data transfer. These are clear advances over the older implantable technology which could only broadcast a fixed identifier, and enable new applications to be realized. As in the 1998 study, the tag was used as an identification device for the University of Reading's intelligent building infrastructure. The author's mobile phone was also augmented with a reader such that only the user with the correct tag could use the phone. In the 1998 study, simple profiles were constructed of users of the building, based on tracking their movements and preferences, which were stored on a central database. Because data can be stored on the latest generation of tags, in a modification, this profile information was stored both in the building's Oracle database and on the implanted HITAG S tag such that the user could enter a new building, which could then access the profile data. Updates to the profile were generated centrally, and written to the tag if it needed updating. While this is seemingly a useful extension to the original system, it comes coupled with new threats, as detailed in the following section.

\subsection{Implantable technology and emerging threats - an experiment}

In 2006, researchers from Vrije Universiteit in Amsterdam demonstrated how commercially available RFID tags could be used to spread malicious computer code [25]. In order to do this the devices required the ability to store data and interact with a potentially vulnerable database system. To demonstrate the concept, a large form factor RFID sticky label tag was infected with a piece of malicious code and used to contaminate a database. Despite the provocative paper title [25] most implantable RFID devices, typically being only readable or of very low data storage capacity, were not vulnerable to this.
Shown in Table 1 is a sample of the contents of the 'PROFILE' table in University of Reading's intelligent building database. In the left column is the unique identifier of each RFID tag or access card (as used by most users of the system). The right column shows the current (old) encoded profile, and the updated (new) encoded profile, as modified by the system, for each user. The system works by reading a user's RFID tag data when they are in proximity of a reader node - this is stored in the 'OldProfile' entry of the database for that tag. This is compared to the updated profile for that user (stored in the 'NewProfile' entry), and if it is different, and it is appropriate to do so, this is written to the tag. While this is a workable implementation, it is susceptible to SQL and script injection attacks, as detailed in [25].

As an experiment, the implanted HITAG $\mathrm{S}$ tag was infected with the malicious code shown in Table 2. Because of the way the malicious code has been written, instead of simply reading data from the implanted tag to store in the database, the system also executes some SQL injection code (see Table 3) which has some dire consequences for the system. The profile data from the infected tag (the initial string of hex encoded data), while likely to be intentionally manipulated as part of the virus, is copied into 'OldProfile' as expected. However, the new profile is also overwritten in the database by a copy of the virus itself. More damagingly, the code designed to ensure that only the database entry for that tag is modified is cleverly commented out by the malicious code. The result is that the virus is copied into the new profile field for all tags, and so any tag subsequently using the system will likely become overwritten and infected. A feature of a computer virus is that is must have the ability to selfreplicate, and this is evident here. Having corrupted the database contents in such a way to allow replication, there is a further 'payload' (some additional malicious activity) associated with the virus. Administration of the database is typically done through a web browser, and once the system is infected the web browser is redirected to another website, denying easy access to rectify the problem. More potentially harmful payloads have previously been demonstrated in [25], including enabling unauthorized system access.

\subsection{Human Enhancement and Bodily Boundaries}

While being a clear demonstration of how implantable devices are becoming more complex, capable and potentially vulnerable [26], being susceptible to a computer virus also raises interesting questions linked to the concept of the body. 
Table 1. An extract of the data stored in the database for four RFID devices, showing the ID number and encoded current and system updated profiles

RFID Tag New Profile / Old Profile as stored in database for a sample of RFID tags

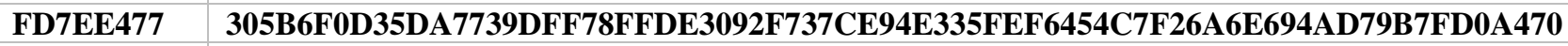
2E5B6F0D35DA7739DFF78FFDE3492F737CE94E335FEF64FFC7F26A6E694AD79B7FD03232

FDC1DB77 49076644BDF82762785A3B0E73C65B9F2653BEEE88991D5311B1AFA4BA332D3CFB36CEF1 1007E644EDF82762785A3B0E73C65BFF2653BEEE88991D531131AFA4BA532D3CFB3635DD

FDCB5377 4B1BA2A1DB9379B837B0C7B1964B8524B1BB2B28BFD9B05679FD8CC516FDB80D4F34AC47 BD1BA221DB9379B837B0C7B1964B8524B14B2B28BFD9B05679FD8CC516FDB80D4F34EE21

FDBD3177 5F0BF32149704E08630F248425FFAF6E86D8A41DFEB43E6A862832AC964EBBBC25BE8EF2 AF0BF321AC704E08630F248425FFAF6E86D8A41D6EB43E6A862832AC964E6BBC25BE9C1C

Table 2. The contents of the infected implanted RFID tag, consisting of malicious computer virus code 41207369676E206F66207468696E677320746F20636F6D65202D20447220476173736F6E',NewProfile=(select SUBSTR(SQL_TEXT,37,209)FROM v\$sqI WHERE INSTR(SQL_TEXT, '<script>window.location=" http://kablamm.com" $<$ /script $>$ ')>0)--

Table 3. The command executed by the Oracle database on reading the infected tag UPDATE USER_PROFILE SET OldProfile='41207369676E206F66207468696E677320746F20636F6D65202D20447 220476173736F6E',NewProfile=(select SUBSTR(SQL_TEXT,37,209)FROM v\$sql WHERE INSTR(SQL_TEXT,' <script>window.location="'http://kablamm.com"</script>')>0)--' WHERE RFID='FDBD3177'

Table 4. The data stored in the database for the same tags as in Table 1 after the database has been compromised by the infected RFID tag

RFID Tag New Profile / Old Profile as stored in database for a sample of RFID tags

FD7EE477 41207369676E206F66207468696E677320746F20636F6D65202D20447220476173736F6E',NewProfile $=($ select SUBSTR $($ SQL_TEXT,37,209)FROM v\$sql WHERE INSTR(SQL_TEXT,' $\langle$ script $>$ window.location="http://kablamm.com" $</$ script $>$ ')>0)-41207369676E206F66207468696E677320746F20636F6D65202D20447220476173736F6E

FDC1DB77 41207369676E206F66207468696E677320746F20636F6D65202D20447220476173736F6E',NewProfile $=\left(\right.$ select SUBSTR(SQL_TEXT,37,209)FROM v\$sql WHERE INSTR(SQL_TEXT, ${ }^{\prime}\langle$ script $>$ window.location="http://kablamm.com" $</$ script $>$ ')>0)-41207369676E206F66207468696E677320746F20636F6D65202D20447220476173736F6E

FDCB5377 41207369676E206F66207468696E677320746F20636F6D65202D20447220476173736F6E',NewProfile

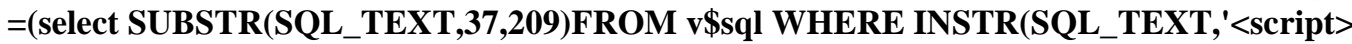
window.location="http://kablamm.com" $</$ script $>$ ')>0)-41207369676E206F66207468696E677320746F20636F6D65202D20447220476173736F6E

FDBD3177 $41207369676 E 206 F 66207468696 E 677320746 F 20636 F 6 D 65202 D 20447220476173736 F 6 E '$ 'NewProfile $=\left(\right.$ select SUBSTR(SQL_TEXT,37,209)FROM v\$sql WHERE INSTR(SQL_TEXT, ${ }^{\prime}\langle$ script $>$ window.location="http://kablamm.com" $</$ script $>$ ')>0)-41207369676E206F66207468696E677320746F20636F6D65202D20447220476173736F6E 
As functions of the body are restored or further enhanced by implanted devices, the boundaries of the body become increasingly unclear. Previous recipients of RFID implants echo the sentiments of many cochlear implant and heart pacemaker users - the implant becomes perceived as being part of the body [27]. That is, what the user understands to be their body includes the technological enhancement. In essence, the boundaries between man and machine simply become theoretical. This development in our traditional notion of what constitutes our body and its boundaries leads to two notable repercussions here. Firstly it becomes possible to talk in terms of a human (albeit a technologically enhanced human) becoming infected by a computer virus. Thus, in that light, the simple experiment presented here has given rise to the world's first human to be infected by a computer virus. Secondly, this development of our concept of the body impacts on certain human rights, in particular the right to bodily integrity. Bodily integrity constitutes a right to do with one's body whatever one wants (a right to self-determination) and it implies the right to prevent one's body from being harmed by others. In this context, a computer virus infecting an implanted device constitutes an infringement on the right to bodily integrity.

A number of wider moral, ethical and legal issues stem from applications of these technologies [28], [29], [30] and it is difficult to foresee the social consequences of adoption long term which may fundamentally change our very conception of self and sense of identity. It is clearly timely to have further and rigorous debate regarding the use of implantable technology in individuals for human enhancement.

\section{Conclusion}

Developments in implantable technologies for enhancement are beginning to redefine our relationship with technology. The changes are not just technological they are driving changes in cultural and social paradigms, and further empowering people to seek new experiences and employ new services. We are already seeing simple technologies such as passive RFID devices being implanted in humans, and this alone introduces challenging questions. In this paper it has been argued that these implantable devices have evolved to the point whereby we should really consider them as simple computers. This radically improved capability over previous generations of the technology has been demonstrated by the infection with a computer virus of an RFID device implanted in a human. Coupled with our developing concept of the body and its boundaries, this has given rise to the world's first human infected with a computer virus.
Technological advancement is a part of our evolution, and the significant next step of forming direct bidirectional links with the human brain is moving inexorably closer. It should not be assumed that since the technology has not yet and may not be perfected that there is no need to address the incipient legal, ethical and social issues that the development of these devices may bring. The basic foundations of advanced implant devices are being developed for clear medical purposes and it is reasonable to assume that few would argue against this progress for such noble, therapeutic causes. Equally, as has been demonstrated by cosmetic surgery, we cannot assume that people will not undergo a procedure because it is highly invasive. So, while we may be some way away, there is clear evidence that devices capable of significant enhancement will become reality, and most probably applied in applications beyond their original purpose. Thus, clear consideration needs to be given now to the fundamental moral, ethical, social, psychological and legal ramifications of such enhancement technologies. It is not too soon to start real debate.

\section{References}

[1] A. Graafstra, "Hands on," IEEE Spectrum, vol. 44(3), 2007, pp. 18-23.

[2] K. Michael and M. G. Michael, "Innovative Automatic Identification and Location-Based Services: From bar codes to chip implants," IGI Global, 2008.

[3] D. A. Kessler, "The basis of the FDA's decision on breast implants," The New England Journal of Medicine, vol. 326, 1992, pp. 1713-1715.

[4] D. Halperin, T. S. Heydt-Benjamin, K. Fu, T. Kohno, and W. H. Maisel, "Security and privacy for implantable medical devices," IEEE Pervasive Computing, vol. 7(1), 2008, pp. 30-39.

[5] F. G. Zeng, "Trends in cochlear implants," Trends in Amplification, vol. 8(1), 2004, pp. 1-34.

[6] P. Hossain, I. W. Seetho, A. C. Browning, and W. M. Amoaku, "Artificial means for restoring vision," British Medical Journal, 330, 2005, pp. 30-33.

[7] J. Olds and P. M. Milner, "Positive reinforcement produced by electrical stimulation of septal area and other regions of rat brain," Journal of Comparative and Physiological Psychology, vol. 47(6), 1954, pp. 419-427. 
[8] S. K. Talwar, S. Xu, E. S. Hawley, S. A. Weiss, K. A. Moxon, and J. K. Chapin, "Rat navigation guided by remote control," Nature, 417, 2002, pp. 37-38.

[9] C. E. Moan and R. G. Heath, "Septal stimulation for the initiation of heterosexual activity in a homosexual male," Journal of Behavior Therapy and Experimental Psychiatry, vol. 3, 1972, pp. 23-30.

[10] J. M. Delgado, "Instrumentation, working hypotheses, and clinical aspects of neurostimulation," Applied Neurophysiology, vol. 40, 1977, pp. 88-110.

[11] T. Wichmann, and M. R. DeLong, "Deep Brain Stimulation for neurologic and neuropsychiatric disorders," Neuron, vol. 52(1), 2006, pp. 197-204.

[12] M. N. Gasson, S. Y. Wang, T. Z. Aziz, J. F. Stein, and K. Warwick, "Towards a demand driven DeepBrain Stimulator for the treatment of movement disorders," 3rd IEE International Seminar on Medical Applications of Signal Processing (MASP2005), London, UK, 3-4 November, 2005, pp. 83-86.

[13] H. Smeding, J. Speelman, M. Koning-Haanstra, P. R. Schuurman, P. Nijssen, T. van Laar, and B. Schmand, "Neuropsychological effects of bilateral STN stimulation in Parkinson disease: A controlled study," Neurology, vol. 66(12), 2006, pp. 1830-1836.

[14] R. Romo, A. Hernandez, A. Zainos, C. D. Brody, and L. Lemus, "Sensing without touching psychophysical performance based on cortical microstimulation," Neuron, 26, 2000, pp. 273-278.

[15] D. Wu, K. Warwick, Z. Ma, M. N. Gasson, J. G. Burgess, S. Pan, T. Z. Aziz, "Prediction of Parkinson's disease tremor onset using a radial basis function neural network based on particle swarm optimization," International Journal of Neural Systems, vol. 20(2), 2010, pp. 109-116.

[16] S. Camporesi, "Oscar Pistorius, Enhancement and Post-humans," Journal of Medical Ethics, vol. 34, 2008, pp. 639.

[17] P. Moore, "Enhancing Me: The Hope and the Hype of Human Enhancement,” John Wiley, 2008.

[18] M. N. Gasson, B. D. Hutt, I. Goodhew, P. Kyberd, and K. Warwick, "Invasive neural prosthesis for neural signal detection and nerve stimulation," International Journal of Adaptive Control and Signal Processing, vol.19(5), 2005, pp. 365-375.
[19] K. Warwick, M. N. Gasson, B. D. Hutt, I. Goodhew, P. Kyberd, B. J. Andrews, P. Teddy, and A. Shad, "The application of implant technology in Cybernetic systems," Archives of Neurology, vol. 60(5), 2003, pp. 1369-1373.

$$
\text { K. Warwick, “I, Cyborg,” Century, } 2002 .
$$

[21] G. R. Cosgrove, "Neuroscience, Brain, and Behavior V: Deep Brain Stimulation," Transcript session 6, June 25, 2004, The President's Council on BioEthics.

http://www.bioethics.gov/transcripts/june04/session6.html

[22] E. M. McGee and G. Q. Maguire, "Becoming borg to become immortal: regulating brain implant technologies," Cambridge Quarterly of Healthcare Ethics, vol. 16(3), 2007, pp. 291-302.

[23] S. Mann, "Wearable Computing: A first step towards personal imaging," IEEE Computer, 30(2), 1997, pp. 25-32.

[24] N, Streitz and P. Nixon, "The Disappearing Computer," Communications of the ACM, vol. 48(3), 2005, pp. 33-35.

[25] M. Rieback, B. Crispo, and A. Tanenbaum, "Is your cat infected with a computer virus?," in Proc. 4th Annual IEEE International Conference on Pervasive Computing and Communication (PERCOM'06), Pisa, Italy, 13-17 March, 2006, pp. 169-179.

[26] W. H. Maisel, T. Kohno, "Improving the security and privacy of implantable medical devices," The New England Journal of Medicine, vol. 362(13), 2010, pp. 1164-1166.

[27] K. Warwick, "Cyborg morals, cyborg values, cyborg ethics," Ethics and Information Technology, vol. 5, 2003, pp. 131-137.

[28] S. Rodotà and R. Capurro, Eds., "Ethical aspects of ICT implants in the human body," Opinion of the European Group on Ethics in Science and New Technologies to the European Commission, 2005, pp. 18$23 \mathrm{D}$.

[29] S. O. Hansson, "Implant ethics," Journal of Medical Ethics, vol. 31, No. 9, 2005, pp. 519-525.

[30] E. Kosta and M. N. Gasson, Eds., "A study on ICT implants," FIDIS, 2008. Available at: http://www.fidis.net/ 